\title{
Ranking of Tornado Outbreaks across the United States and Their Climatological Characteristics
}

\author{
Christopher M. Fuhrmann,* Charles E. Konrad II, And Margaret M. Kovach \\ Southeast Regional Climate Center, Department of Geography, University of North Carolina at Chapel Hill, \\ Chapel Hill, North Carolina \\ JORDAN T. MCLEOD \\ Climatology Research Laboratory, Department of Geography, The University of Georgia, Athens, Georgia \\ WILLIAM G. SCHMITZ \\ Southeast Regional Climate Center, Department of Geography, University of North Carolina at Chapel Hill, \\ Chapel Hill, North Carolina \\ P. GRADY DIXON \\ Department of Geosciences, Mississippi State University, Mississippi State, Mississippi
}

(Manuscript received 30 October 2013, in final form 14 March 2014)

\begin{abstract}
The calendar year 2011 was an extraordinary year for tornadoes across the United States, as it marked the second highest annual number of tornadoes since 1950 and was the deadliest tornado year since 1936. Most of the fatalities in 2011 occurred in a series of outbreaks, highlighted by a particularly strong outbreak across the southeastern United States in late April and a series of outbreaks over the Great Plains and Midwest regions in late May, which included a tornado rated as a category 5 event on the enhanced Fujita scale (EF5) that devastated the town of Joplin, Missouri. While most tornado-related fatalities often occur in outbreaks, very few studies have examined the climatological characteristics of outbreaks, particularly those of varying strength. In this study a straightforward metric to assess the strength, or physical magnitude, of tornado outbreaks east of the Rocky Mountains from 1973 to 2010 is developed. This measure of outbreak strength, which integrates the intensity of tornadoes [Fujita (F)/EF-scale rating] over their distance traveled (pathlength), is more highly correlated with injuries and fatalities than other commonly used variables, such as the number of significant tornadoes, and is therefore more reflective of the potential threat of outbreaks to human life. All outbreaks are then ranked according to this metric and their climatological characteristics are examined, with comparisons made to all other tornadoes not associated with outbreaks. The results of the ranking scheme are also compared to those of previous studies, while the strongest outbreaks from 2011 are ranked among other outbreaks in the modern record, including the April 1974 Super Outbreak.
\end{abstract}

* Current affiliation: Department of Geosciences, Mississippi
State University, Mississippi State, Mississippi.

Corresponding author address: Dr. Christopher M. Fuhrmann, Southeast Regional Climate Center, Dept. of Geography, University of North Carolina at Chapel Hill, Saunders Hall, Campus Box 3220, Chapel Hill, NC 27599-3220.

E-mail: fuhrmann@unc.edu

\section{Introduction}

The calendar year 2011 was an extraordinary year for tornadoes across the United States. Nearly 1700 tornadoes were confirmed, making it the second highest annual total since 1950 (NOAA 2012). Most significantly, there were an estimated 553 tornado-related fatalities in 2011, resulting in the deadliest tornado year since 1936 and second deadliest since 1875 (SPC 2013). Most of 


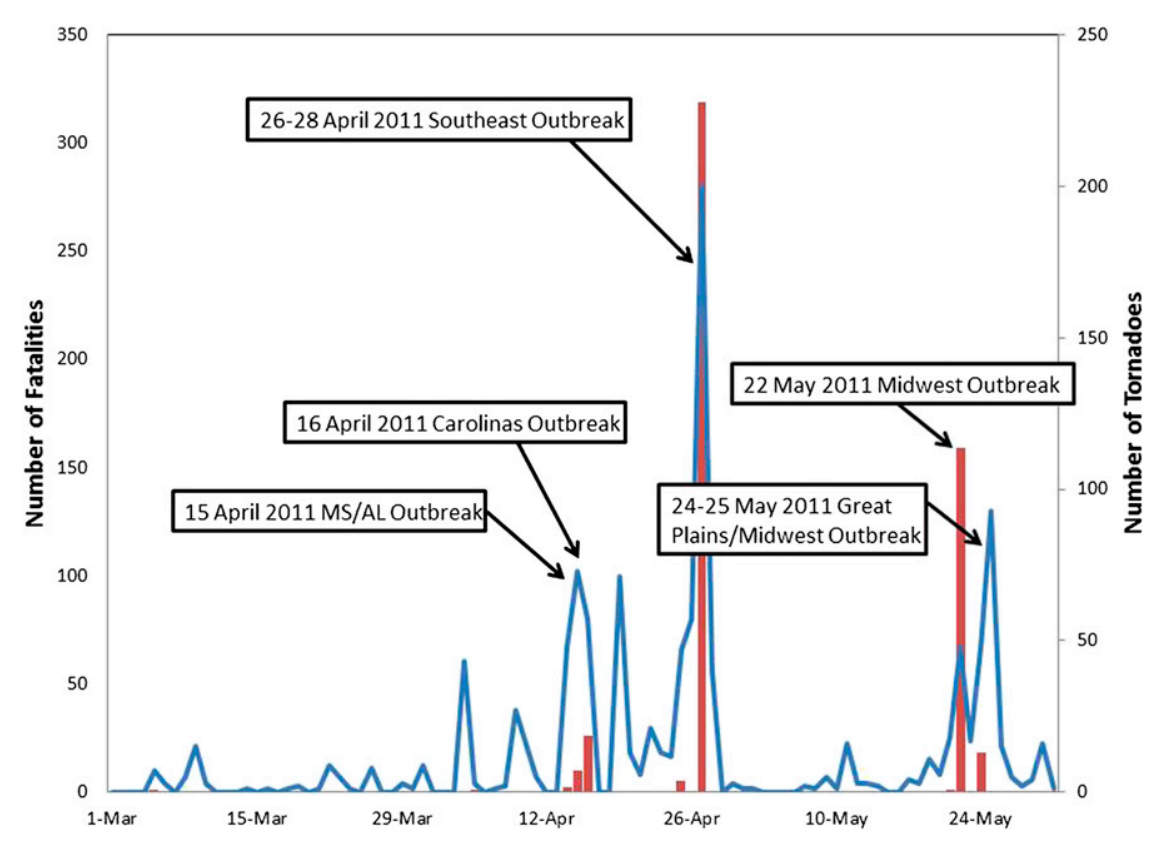

FIG. 1. Daily counts of tornadoes (blue line) and tornado-related fatalities (red bars) across the United States from 1 Mar to 31 May 2011. Five notable tornado outbreaks during this period are identified. These outbreaks accounted for over $95 \%$ of all tornado-related fatalities in 2011.

these fatalities occurred in what are commonly referred to as outbreaks (Fig. 1). An outbreak can be broadly defined as a group of tornadoes associated with a single synoptic-scale weather system (Shafer and Doswell 2010), though specific criteria, such as the number of tornadoes, typically vary from one study to the next (Doswell et al. 2006; Verbout et al. 2006). Because outbreaks involve multiple tornadoes, some of which may be violent and long track, they are more likely to affect populated areas, increasing the likelihood of fatalities (Brooks 2004). Galway (1977) noted that $73 \%$ of all tornado-related fatalities in the United States from 1952 to 1973 occurred in outbreaks with at least 10 tornadoes, while Schneider et al. (2004) found that tornado outbreak days from 1875 to 2003 accounted for over $80 \%$ of all tornado-related fatalities. With increasing population density and urban sprawl, more people will likely be exposed to future tornado outbreaks.

While the overall number of tornadoes is sometimes used to define an outbreak, it is recognized that the intensity of the tornadoes (i.e., Fujita-scale, or F-scale rating; changed to enhanced Fujita, or EF scale, in 2007) is also important in assessing the physical magnitude, or strength, of an outbreak (Johns and Sammler 1989; Verbout et al. 2006) and the potential for significant societal impacts (Brooks 2004). As a result, the term outbreak is often used to refer to major events involving several strong to violent tornadoes that occur over a broad area (e.g., several states) for at least 1 day (e.g., the April 1974 Super Outbreak, the November 1992 "Widespread" outbreak). Additionally, there are cases where several strong to violent tornadoes (F/EF2 and greater) are observed in an unbroken sequence over several consecutive days, sometimes over the same region. For example, Hamill et al. (2005) documented a case where strong tornado activity was reported for nine consecutive days in early May 2003 across the Great Plains and Midwest regions. However, strong (F/EF2 and F/EF3) and violent (F/EF4 and F/EF5) tornadoes compose only about $18 \%$ and $1 \%$ of all tornadoes in a given year across the United States, respectively (Doswell et al. 2012). Therefore, the term outbreak can also be applied more broadly to groups of mostly weak tornadoes ( $\mathrm{F} / \mathrm{EF} 0$ and $\mathrm{F} / \mathrm{EF} 1)$, as well as those that occur over generally small areas (e.g., a county) and/or over relatively short periods of time (e.g., a few hours) (Doswell et al. 2006), particularly if they are associated with significant societal impacts.

Despite the dangers imposed by tornado outbreaks, we are unaware of any previous attempts to examine the climatological distribution and characteristics of outbreaks according to their physical magnitude. Galway (1977) examined the climatology of tornado outbreaks in the United States from 1952 to 1973, but did not evaluate their intensity. Johns and Sammler (1989) developed a climatology that considered violent outbreaks, which were defined by the occurrence of at least 
one F4 tornado. In addition, several studies have calculated the strength of tornado outbreaks through various ranking schemes (e.g., Thompson and Vescio 1998; Doswell et al. 2006; Forbes 2006; Shafer and Doswell 2011), but none have extensively assessed the climatological aspects of the strength of outbreaks. To help fill this gap, we develop a straightforward metric to assess the strength of tornado outbreaks across the United States. This metric is different from those used in prior studies in that it focuses exclusively on the physical strength of outbreaks, which is defined by the sum of the work done by all tornadoes in the outbreak (i.e., the force of the winds associated with a tornado integrated across the area that it impacts). Using this metric, we carry out the following:

1) rank all outbreaks and examine their climatological and geographical characteristics,

2) compare the characteristics of outbreak tornadoes with those tornadoes not connected with outbreaks,

3) assess how the results of our ranking scheme compare to those of previous studies, and

4) ascertain how the strongest outbreaks from 2011 (Fig. 1) rank among other notable outbreaks in the modern era (i.e., since the early 1970s).

Although physical magnitude is just one measure of the significance of a given outbreak (Stimers 2012), it is a useful starting point in assessing the potential lethality and overall impact of an outbreak on society.

\section{Development of outbreak definition and ranking scheme}

\section{a. Tornado data}

Tornado outbreaks were constructed from individual tornado reports obtained from the Storm Prediction Center (SPC) using the online SeverePlot tool (http:// www.spc.noaa.gov/climo/online/sp3/plot.php) for the period 1973-2010. Only tornadoes reported east of the Rocky Mountains were obtained. Each tornado record in the dataset includes the time of occurrence, location (latitude and longitude) of touchdown and liftoff, path width, pathlength, F/EF-scale rating, number of fatalities, and number of injuries. Official tornado reports from 2011 were made publicly available by the SPC in June 2012 and were used to identify the deadliest outbreaks from 2011 and assess their ranking relative to all outbreaks identified from 1973 to 2010.

Although the dataset obtained from the SPC represents the official record of tornado activity in the United States, there are some limitations and possible sources of error. These include inconsistencies in data collection and verification procedures, improvements in monitoring technology (e.g., Doppler radar), and the implementation of storm spotter networks [see Verbout et al. (2006) for a detailed discussion of the evolution of the U.S. tornado database]. An important feature of the tornado database is a doubling of tornado reports from the 1950 s to the 2000s. Most of this increase is due to the more frequent reporting of weak (i.e., F/EF0) tornadoes over time (Verbout et al. 2006). By comparison, the reporting of $\mathrm{F} / \mathrm{EF} 1$ and greater tornadoes has remained fairly stable and is therefore more representative of the trend in tornado activity since the 1950s (Brooks and Doswell 2001; Verbout et al. 2006). However, systematic changes in the collection of tornado data have also taken place and these changes have resulted in secular trends in the SPC dataset. Most notably, there has been a significant decrease in the number of F/EF2 and greater tornadoes since the early 1970s (Verbout et al. 2006). This coincided with a shift in responsibility for reporting tornadoes from the federal state climatologist to the National Weather Service (NWS) (Brooks and Craven 2002). Prior to this shift, tornadoes were rated using archived newspaper accounts and photographs, which tended to emphasize higher degrees of damage and devastation (Doswell et al. 2009). Therefore, as in Brooks and Craven (2002) and Coleman and Dixon (2014), we chose 1973 as a start date to limit the influence of known secular trends in the tornado data, though by doing so our sample size was reduced by approximately $30 \%$ compared to the period beginning in 1954. However, it has been suggested that in order to obtain the most reliable tornado record possible for analysis, it is better to limit the influence of secular trends, even at the expense of a larger sample size (Doswell 2007).

There are also some potential errors in the use of the F scale to rate tornado intensity due to the fact that the F scale uses damage indicators from built structures to estimate the maximum wind speed associated with the tornado (Doswell et al. 2009). Relating the structural damage from a tornado to a given wind speed has been the subject of much study, and in February 2007 the F scale was replaced with the EF scale to more accurately relate the damage from a wider variety of structures to the estimated maximum wind speed of the tornado (Doswell et al. 2009). A recent assessment of tornadoes rated using the EF scale suggests an increase in assessed damage, with more EF1 and EF2 tornadoes and fewer EF0 tornadoes compared to the rest of the NWS modernization period beginning in the mid-1990s (Edwards and Brooks 2010). The impact of the EF scale on the long-term climatology of tornadoes, however, remains unclear (Edwards et al. 2013). In addition, tornadoes that strike less-populated or rural areas are less likely to 
produce any structural damage. With fewer damage indicators, the intensity of these tornadoes may be underestimated (Boruff et al. 2003). Despite some uncertainty in the relationship between damage and wind speed, and the likely underestimation of tornado strength in rural areas, any systematic errors in the assignment of an F/EFscale rating should not be detectable with a large sample size (Brooks 2004; Doswell et al. 2009).

\section{b. Methods}

For this study, we defined a tornado outbreak as a sequence of six or more tornadoes with an F/EF-scale rating of 1 or greater with no more than a 6 -h period between consecutive tornadoes in the sequence. The stipulation that an outbreak must contain at least 6 tornadoes is consistent with criteria used by Pautz (1969) and Grazulis (1993) and is less restrictive than the 10 tornadoes required by Galway (1977), thereby allowing for a wider range of outbreak sizes to be examined. Only tornadoes with an F/EF-scale rating of 1 and greater were considered in the outbreak definition to account for the increase in F/EF0 tornadoes over time and potential bias in the frequency and size of outbreaks toward the latter part of the study period (Verbout et al.2006). Also, F/EF0 tornadoes have been associated with only $0.5 \%$ and $1.2 \%$ of the total number of fatalities and injuries from 1973 to 2010, respectively. The tornado-free period of $6 \mathrm{~h}$ or greater was adopted from Forbes (2006) and Grazulis (1993). We chose to use this criterion as opposed to the "tornado day" used by Doswell et al. (2006) and Verbout et al. (2006) in order to account for tornado sequences of varying durations and starting and ending times. Due to the large number of outbreaks identified in this study (over 800), we did not examine corresponding synoptic surface analyses to determine if any tornadoes in an outbreak were geographically and/or meteorologically disconnected. Doswell et al. (2006) found that this was not an issue in classifying major outbreaks; however, we cannot guarantee that all tornado tracks associated with a given outbreak are part of the same synoptic-scale weather system.

One of the main objectives of this study is to develop and apply a metric for assessing the strength or magnitude of tornado outbreaks. The property damage and casualties from a given tornado can be related to its strength and the area that it impacts (i.e., the pathlength multiplied by the path width) (Thompson and Vescio 1998). The strength of a given tornado is typically associated with its assigned F/EF-scale rating. The force of the associated winds can be estimated from the median wind speed that is related to the tornado's F/EF-scale rating. If this force is multiplied by the pathlength or the area impacted by the tornado, then an estimate of the amount of work done by the tornado is provided. By summing these values across all of the tornadoes in an outbreak, an estimate of outbreak magnitude or strength is generated (Doswell et al. 2006; Shafer and Doswell 2010, 2011).

In this study, we developed two metrics for the strength of the tornadoes in each outbreak: 1) hectopascal milesthe estimated pressure exerted by the median wind speed (determined from the F/EF-scale rating of each tornado) multiplied by the pathlength of the tornado, and 2) Fujita miles - the F/EF-scale rating of the tornado multiplied by its pathlength. Hectopascal and Fujita miles were summed across the tornadoes in each outbreak to provide an estimate of its strength. These two measures were found to be highly correlated with each other $(r=0.98)$ across the population of tornado outbreaks in this study. Therefore, we chose to use the simpler measure, Fujita miles, since it may be more easily communicated to the public. In addition, because the F/EF-scale rating is used in the calculation, F/EF0 tornadoes are not assigned any Fujita miles (i.e., pathlength multiplied by zero), which eliminates bias in the measure of outbreak intensity over the latter part of the study period due to the increased reporting of $\mathrm{F} / \mathrm{EF} 0$ tornadoes.

To determine if our outbreak criteria and measure of outbreak strength exhibited any marked trends over time, we fitted regression lines to the annual number of outbreaks and cumulative number of Fujita miles. As in Doswell et al. (2006), we used an extended period for which we had tornado data, which in this case went back to 1954. No discernible trend was noted in the number of outbreaks, while a slight downward but statistically insignificant trend was noted in the number of outbreak Fujita miles (Fig. 2). Considerable interannual variability was apparent in both time series. Therefore, the detrending process (see Doswell et al. 2006) was not implemented in this study. When examined by subperiods, the annual average number of Fujita miles was $16 \%$ higher from 1954 to 1972 compared to the period from 1973 to 2010, while the annual average number of F/EF1 (F/EF2) and greater tornadoes was $10 \%$ (41\%) higher during the pre1973 period. These results further support the use of 1973 as the start date for the analyses.

One issue with the use of data from the SPC tornado record is that only the maximum $\mathrm{F} / \mathrm{EF}$-scale rating is provided for each tornado. Surveys conducted after a tornado often reveal much variability in the degree of damage (and therefore the estimated wind speed) along the path. Therefore, the maximum F/EF-scale rating will often overestimate the true intensity of a tornado integrated along its entire path (Doswell and Burgess 1988). To account for this, we obtained data for individual segments of a sample of tornadoes from the National Weather Service's Performance Management and Data 

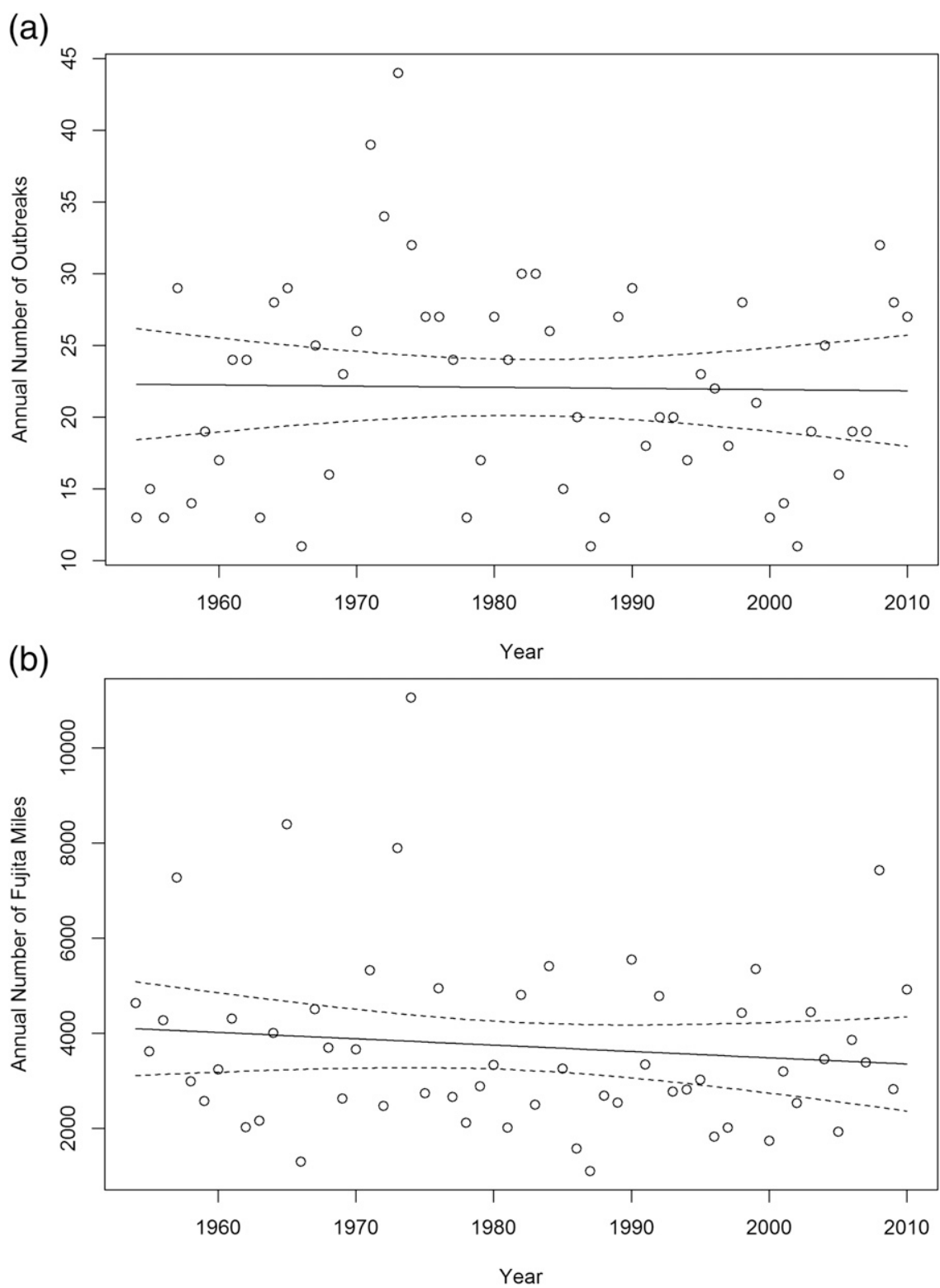

FIG. 2. Plots of the annual number of (a) tornado outbreaks and (b) Fujita miles $(1 \mathrm{mi}=$ $1.6 \mathrm{~km}$ ) across the eastern two-thirds of the United States from 1954 to 2010 . The solid line in each figure represents a linear regression fit to the data and the two dotted lines represent the $95 \%$ confidence intervals for the regression coefficients.

Verification website (http://verification.nws.noaa.gov/ services/public/index.aspx; Table 1). Tornadoes associated with outbreaks from 2006 to 2011 were randomly selected from the database and categorized by maximum F/EF-scale rating. To minimize potential geographical reporting biases, tornadoes were sampled from 18 states across the eastern United States. For each tornado, we calculated the ratio of Fujita miles summed across each segment of the tornado track to the number of Fujita miles using the maximum $\mathrm{F} / \mathrm{EF}$-scale rating. This ratio was then multiplied by the number of Fujita miles using the maximum $\mathrm{F} / \mathrm{EF}$-scale rating to determine the number of adjusted Fujita miles (AFMs) for each tornado. Finally, a mean adjustment factor for each F/EF-scale rating was computed by averaging all of the individual ratios across the sample of tornadoes (Table 1). This adjustment factor was then applied to all tornadoes in the study from 1973 to 2010, as well as those from 2011.

While path width has been used in other measures of outbreak intensity [e.g., the Forbes impact index; 
TABLE 1. Mean adjusted F/EF-scale ratings with standard deviation and sample size of tornadoes used to calculate the adjusted ratings.

\begin{tabular}{cccc}
\hline \hline $\begin{array}{c}\text { F/EF-scale } \\
\text { rating }\end{array}$ & $\begin{array}{c}\text { Mean adjusted } \\
\text { F/EF-scale rating }\end{array}$ & Std dev $( \pm 1)$ & $\begin{array}{c}\text { Sample } \\
\text { size }\end{array}$ \\
\hline 1 & 0.981 & $0.912-1.050$ & 60 \\
2 & 1.818 & $1.456-2.180$ & 32 \\
3 & 2.769 & $2.514-3.024$ & 31 \\
4 & 3.544 & $3.064-4.024$ & 26 \\
5 & 4.430 & $3.830-5.030$ & 4 \\
\hline
\end{tabular}

Forbes (2006)] and the destruction potential index (DPI; Thompson and Vescio 1998), we chose not to use path width due to inconsistent reporting in the SPC records. Until 1995, only the mean path width along the tornado track was reported. Since 1995, the reported path width has corresponded to the maximum width recorded along the track. As a result, Brooks (2004) states that the use of path width is of limited value in assessing tornado intensity. Indeed, our measure of outbreak strength, which integrates the intensity of tornadoes (F/EF-scale rating) over their distance traveled (pathlength), is more highly correlated with injuries and fatalities than other commonly used variables, including the DPI (Table 2) and is, therefore, more reflective of the potential threat of outbreaks to human life.

\section{Climatology and geographical characteristics of tornado outbreaks}

\section{a. General characteristics}

A total of 846 tornado outbreaks were identified across the eastern two-thirds of the United States from 1973 to 2010. These outbreaks accounted for more than half of all F/EF1 and greater tornadoes, $83 \%$ of all violent tornadoes (F/EF4 and F/EF5), and 79\% of all tornadorelated fatalities (Table 3). Outbreaks ranged in strength from 0.8 AFMs to over 7000 AFMs; however, more than half of the outbreaks had fewer than 100 AFMs and more than $90 \%$ exhibited fewer than 500 AFMs (Table 3). Figure 3 displays the rankings, or distribution, of all outbreaks according to their AFMs and reveals that the

TABLE 2. Correlation coefficients between different tornado outbreak variables and the number of fatalities and injuries from 1973 to 2010. All coefficients are significant at the $99 \%$ confidence level.

\begin{tabular}{lcc}
\hline \hline \multicolumn{1}{c}{ Variable } & Fatalities & Injuries \\
\hline Adjusted Fujita miles (AFMs) & 0.80 & 0.81 \\
Destruction potential index (DPI) & 0.70 & 0.68 \\
No. of significant tornadoes ( $\geq$ F/EF2) & 0.66 & 0.69 \\
No. of tornadoes $\geq$ F/EF1 & 0.49 & 0.52 \\
\hline
\end{tabular}

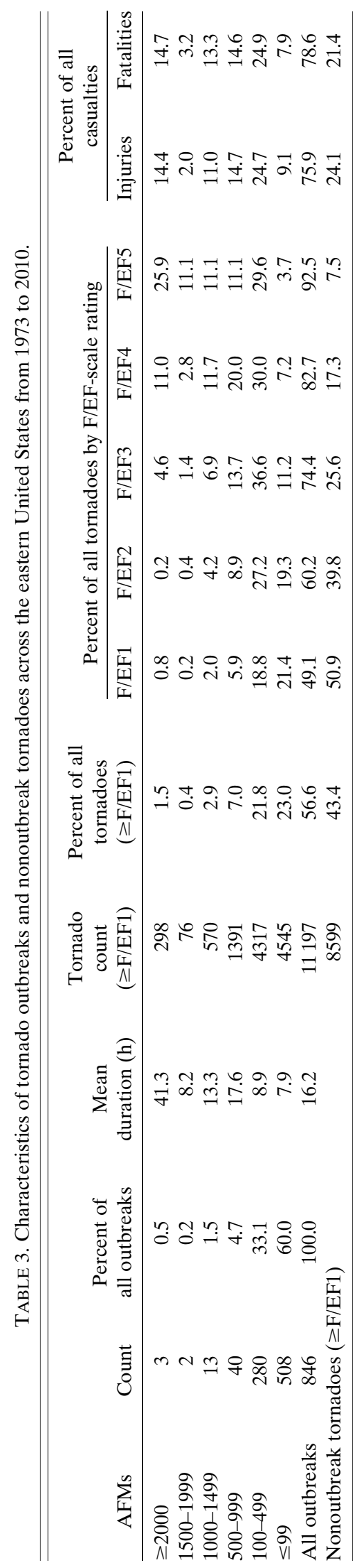




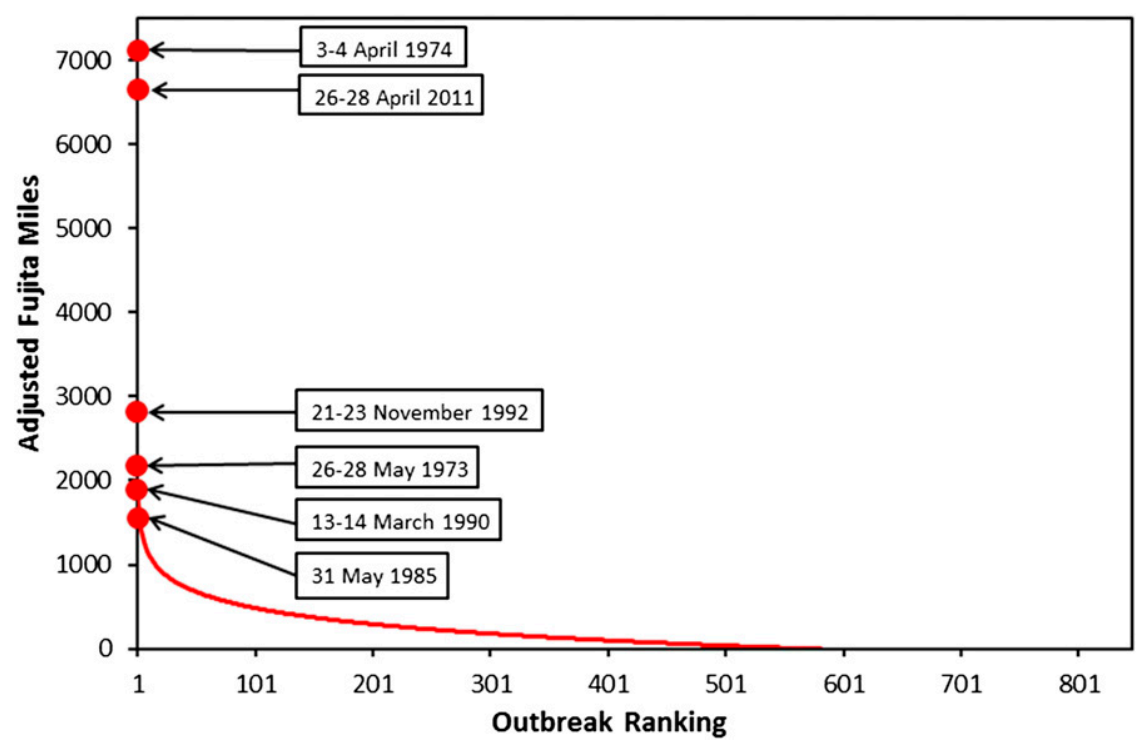

FIG. 3. Distribution of tornado outbreaks across the eastern two-thirds of the United States from 1973 to 2010 by AFMs. The dates and relative rankings of the top-five outbreaks during this period are provided, as well as the relative ranking of the 26-28 Apr 2011 outbreak.

top $2 \%$ of all outbreaks (i.e., those with at least 1000 AFMs) exhibited a range of over 6000 AFMs, while the bottom $50 \%$ of all outbreaks exhibited a range of only 100 AFMs. The range in strength between outbreaks was greatest among the top five outbreaks (i.e., those with at least 1500 AFMs), with the strongest outbreak-the April 1974 Super Outbreak-approximately 2.5 times stronger than the second and third strongest outbreaks (excluding the April 2011 outbreak). The distribution of outbreak rankings here is qualitatively similar to the distribution shown in Doswell et al. (2012).

The strength of the outbreaks was positively related to their duration, with the stronger outbreaks generally exhibiting the longest durations (Table 3). The mean duration among all outbreaks was less than $24 \mathrm{~h}$, with the bottom $90 \%$ of outbreaks (i.e., those with fewer than 500 AFMs) lasting less than $12 \mathrm{~h}$. In contrast, the top three outbreaks exhibited a mean duration of over $40 \mathrm{~h}$. The top three outbreaks also accounted for approximately $15 \%$ of all tornado-related fatalities, despite accounting for only $1.5 \%$ of all tornadoes, while the 18 outbreaks with at least 1000 AFMs were responsible for over 30\% of all tornado-related fatalities and over $25 \%$ of all tornado-related injuries. Moreover, the fatalities from these outbreaks were typically distributed among several tornadoes, with approximately $40 \%$ of the fatalities occurring in outbreaks with at least six killer tornadoes. In contrast, over $90 \%$ of the fatalities connected with very weak outbreaks (i.e., those with fewer than 100 AFMs) were distributed among just one or two tornadoes per outbreak.

\section{b. Spatial patterns}

To visualize the spatial patterns of outbreak and nonoutbreak tornadoes, we employed kernel density estimation, which has been used routinely in tornado climatologies and tornado risk assessments (see Coleman and Dixon 2014). Similar to previous studies (e.g., Dixon et al. 2011; Smith et al. 2012; Coleman and Dixon 2014), we used the Epanechnikov quadratic kernel probability density function. To determine the most appropriate kernel radius, we used the global Moran's $I$ index (O'Sullivan and Unwin 2003) to assess the spatial autocorrelation of tornado paths for strong outbreaks (i.e., at least $500 \mathrm{AFMs}$ ), weak outbreaks (i.e., less than $500 \mathrm{AFMs}$ ), and nonoutbreak tornadoes (Table 4). A minimum applicable radius was chosen according to the smallest statistically significant value that displayed a peak in spatial autocorrelation (Dixon et al. 2014). To be a valid test of spatial autocorrelation, the radius in question must be large enough to ensure that every tornado path is within the range of at least one other path (i.e., neighbor). The relatively small sample size of

TABLE 4. Results of Moran's I spatial autocorrelation analysis.

\begin{tabular}{cccc}
\hline Kernel radius $(\mathrm{km})$ & Moran's $I$ & $Z$ score & $p$ value \\
\hline $350^{*}$ & 0.0109 & 6.94 & $<0.0001$ \\
$360^{*}$ & 0.0100 & 6.56 & $<0.0001$ \\
$370^{*}$ & 0.0102 & 6.93 & $<0.0001$ \\
380 & 0.0099 & 6.93 & $<0.0001$ \\
390 & 0.0097 & 7.04 & $<0.0001$ \\
\hline
\end{tabular}

* Radii that left some tornado paths without neighbors. 
(a)

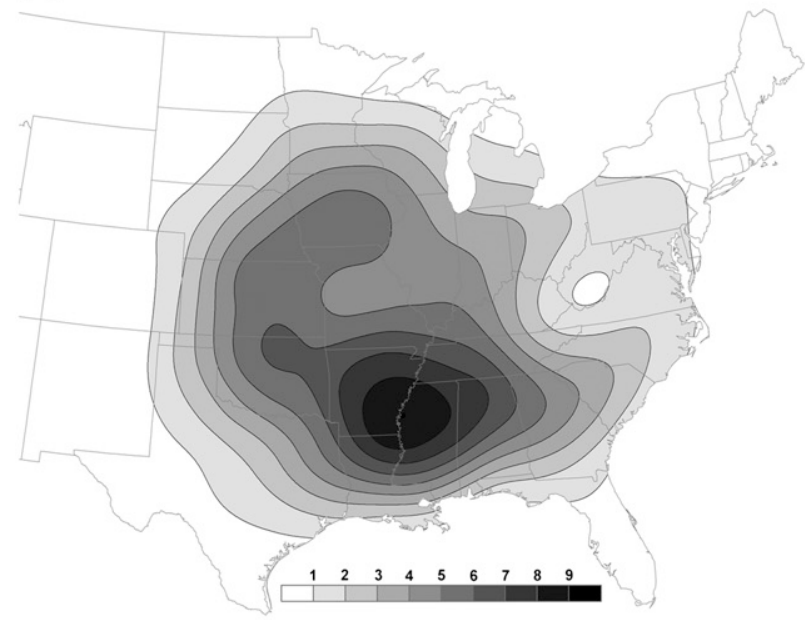

(c)

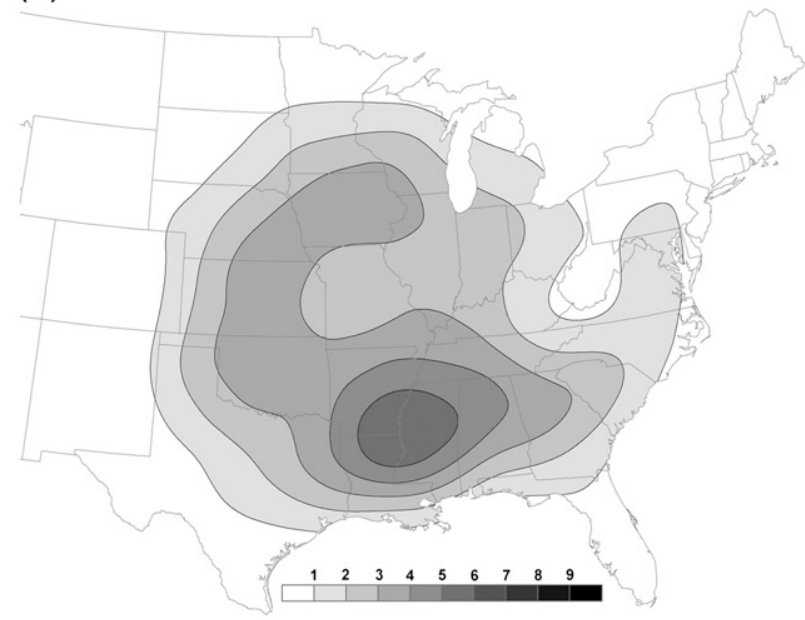

(b)

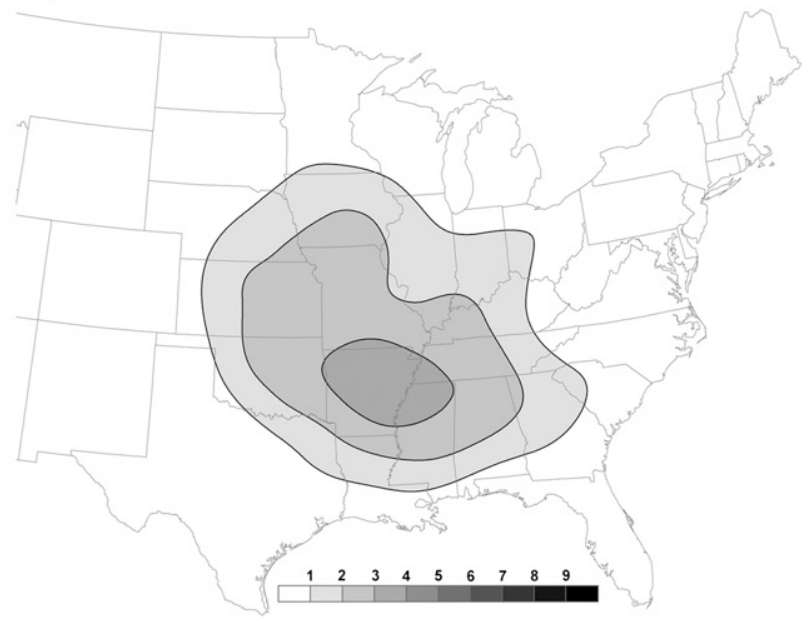

(d)

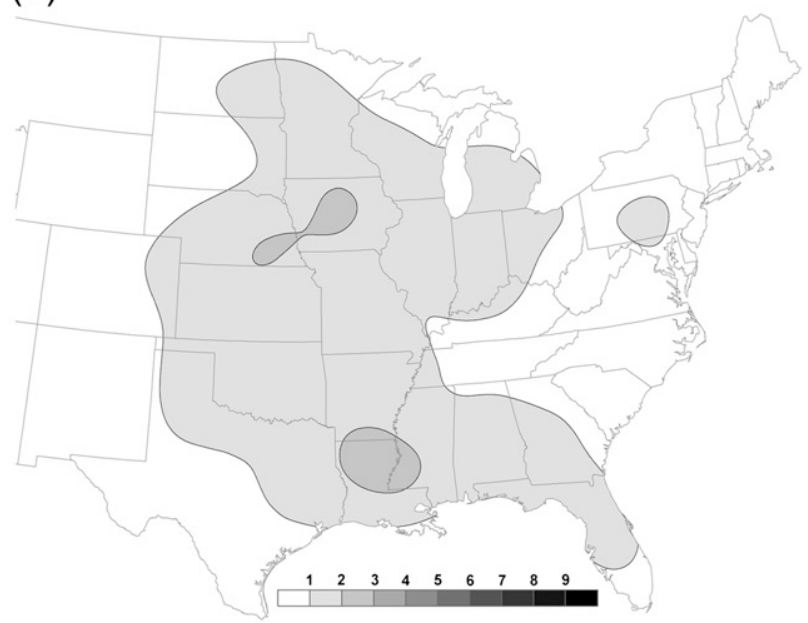

FIG. 4. Kernel density estimations of the average annual pathlength $(\mathrm{km})$ of tornadoes associated with (a) all outbreaks, (b) strong outbreaks, (c) weak outbreaks, and (d) nonoutbreaks passing within $40 \mathrm{~km}$ of a point from 1973 to 2010.

outbreak tornadoes with at least 500 AFMs (see Table 3), and resulting path density, required a smoothing radius of at least $380 \mathrm{~km}$ to account primarily for several paths that were rather isolated from larger clusters. For consistency, this same radius was used to calculate average probabilities of exposure to all outbreak and nonoutbreak tornadoes over the eastern two-thirds of the United States. The resulting probabilities are displayed visually as the average annual pathlength of tornadoes within $40 \mathrm{~km}$ of any point, which provides a robust assessment of tornado risk by defining spatial patterns of exposure [i.e., the area integrated along the pathlength; Dixon et al. (2011); Coleman and Dixon (2014)] rather than tornado frequency or touchdown point (Brooks et al. 2003). Moreover, this method matches the spatial domain across which probabilistic forecasts of tornado risk are issued by the SPC (Kay and Brooks 2000).
The map of outbreak risk reveals a broad area across the Great Plains, Midwest, and Southeast, with an average annual pathlength of at least $4 \mathrm{~km}$ (Fig. 4a). Within this broad area is a region of greater pathlength (at least $6 \mathrm{~km}$ ) that extends from northeastern Alabama through the traditional "Tornado Alley" region of the Great Plains. This region coincides roughly with the area of greatest risk from significant tornadoes (i.e., F/EF2 and greater) identified by Concannon et al. (2000), as well as the centroid locations of major outbreak days identified by Mercer et al. (2009). The area of greatest annual outbreak pathlength (at least $8 \mathrm{~km}$ ) is centered along part of the lower Mississippi River valley and extends from near Little Rock, Arkansas, to Jackson, Mississippi, and south toward Monroe, Louisiana.

The geographic distribution of average annual pathlength is more spatially compact among strong outbreaks 


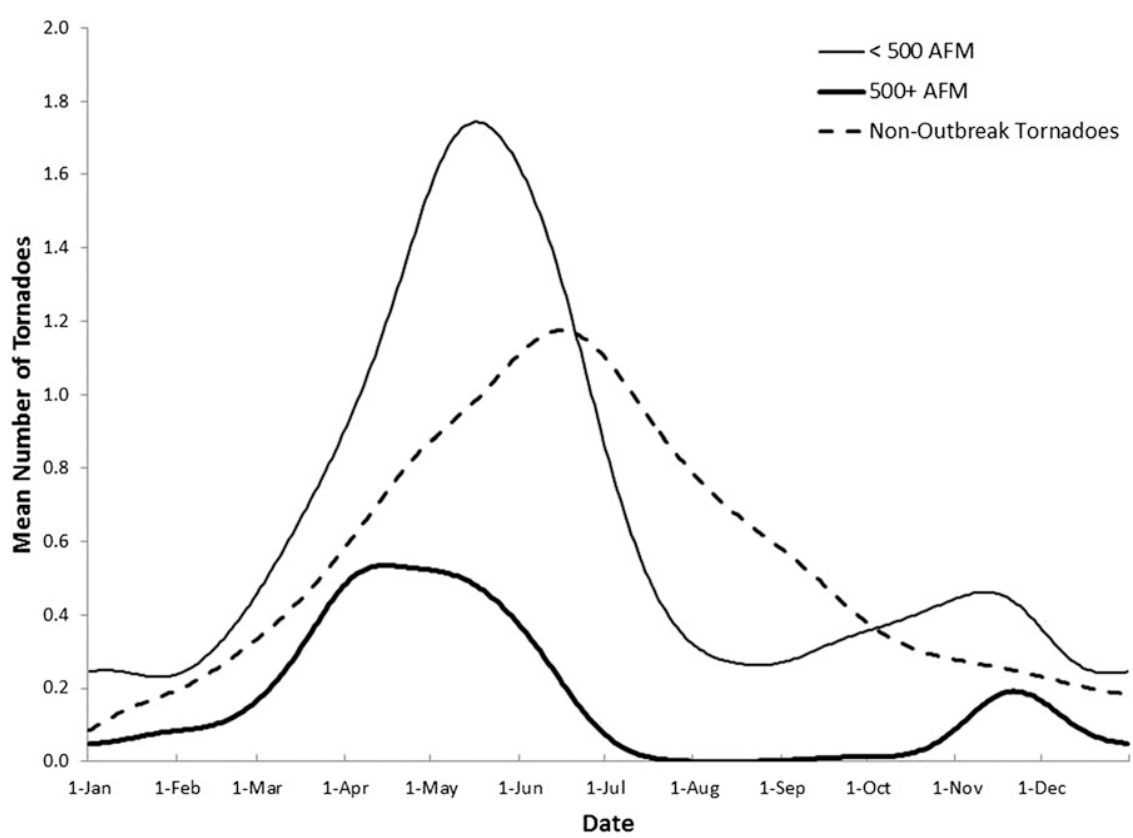

FIG. 5. Daily distribution of tornadoes associated with strong outbreaks, weak outbreaks, and nonoutbreaks across the eastern two-thirds of the United States from 1973 to 2010.

(i.e., those with at least $500 \mathrm{AFMs}$ ) relative to weak outbreaks (i.e., those with less than 500 AFMs) (Figs. 4b,c). In addition, the greatest annual risk of strong tornado outbreaks is lower (at least $3 \mathrm{~km}$ ) than the risk from weak outbreaks (at least $5 \mathrm{~km}$ ) and located slightly farther north and west across much of Arkansas into southwestern Tennessee. The lower maximum risk values among strong outbreaks may be related to their lower frequency compared to weak outbreaks, as they composed only $7 \%$ of all outbreaks and roughly $20 \%$ of all outbreak tornadoes (Table 3). Because significant, longtrack tornadoes occur less frequently across Florida and New England, these areas do not appear on any of the outbreak risk maps, which have a minimum contour interval of $1 \mathrm{~km}$ for their average annual pathlength. Hagemeyer (1997) suggested that tornado outbreaks across Florida are distinct from outbreaks across the rest of the United States, not only in terms of meteorological patterns but also in the strength and pathlength of the tornadoes. For example, only 1 of the 22 outbreaks identified by Hagemeyer (1997) across the Florida Peninsula from 1973 to 1994 exhibited greater than 100 AFMs.

By comparison, the map of nonoutbreak tornado risk reveals a broad area of at least $1 \mathrm{~km}$ of average annual pathlength that generally coincides with the broad area of risk associated with outbreak tornadoes found across the Great Plains and Midwest (Fig. 4d). The area of risk also extends into the central peninsula of Florida. In contrast, the average annual pathlength of nonoutbreak tornadoes is less than $1 \mathrm{~km}$ across much of the Southeast and mid-Atlantic regions, as well as parts of the Ohio River and Tennessee River valleys. Therefore, these regions do not appear on the nonoutbreak tornado risk map. The areas of greatest annual nonoutbreak pathlength (at least $2 \mathrm{~km}$ ) are found across southeastern Nebraska and central Iowa as well as across much of northern Louisiana, extreme southern Arkansas, and southwestern Mississippi. If we overlay the risk maps of outbreak and nonoutbreak tornadoes (not shown), an area of maximum overall risk is found across the lower Mississippi River valley and includes parts of southeastern Arkansas, northeastern Louisiana, and western Mississippi.

\section{c. Temporal patterns}

The annual cycle of outbreak and nonoutbreak tornadoes was determined by smoothing the daily frequency of tornadoes over the 38-yr record using a 15-day Gaussian smoother [Eq. (1) in Brooks et al. (2003)]. Because the raw frequency of tornadoes exhibits considerable day-to-day variability, smoothing is necessary to obtain a more reasonable estimation of the true underlying distribution of tornadoes throughout the year (Brooks et al. 2003). The resulting smoothed frequencies were then converted to average annual frequencies of outbreak and nonoutbreak tornadoes.

The mean frequency of weak outbreak tornadoes reveals a well-defined peak around mid-May, with a smaller, secondary peak in mid-November (Fig. 5). The mean frequency of strong outbreak tornadoes reveals a broader 

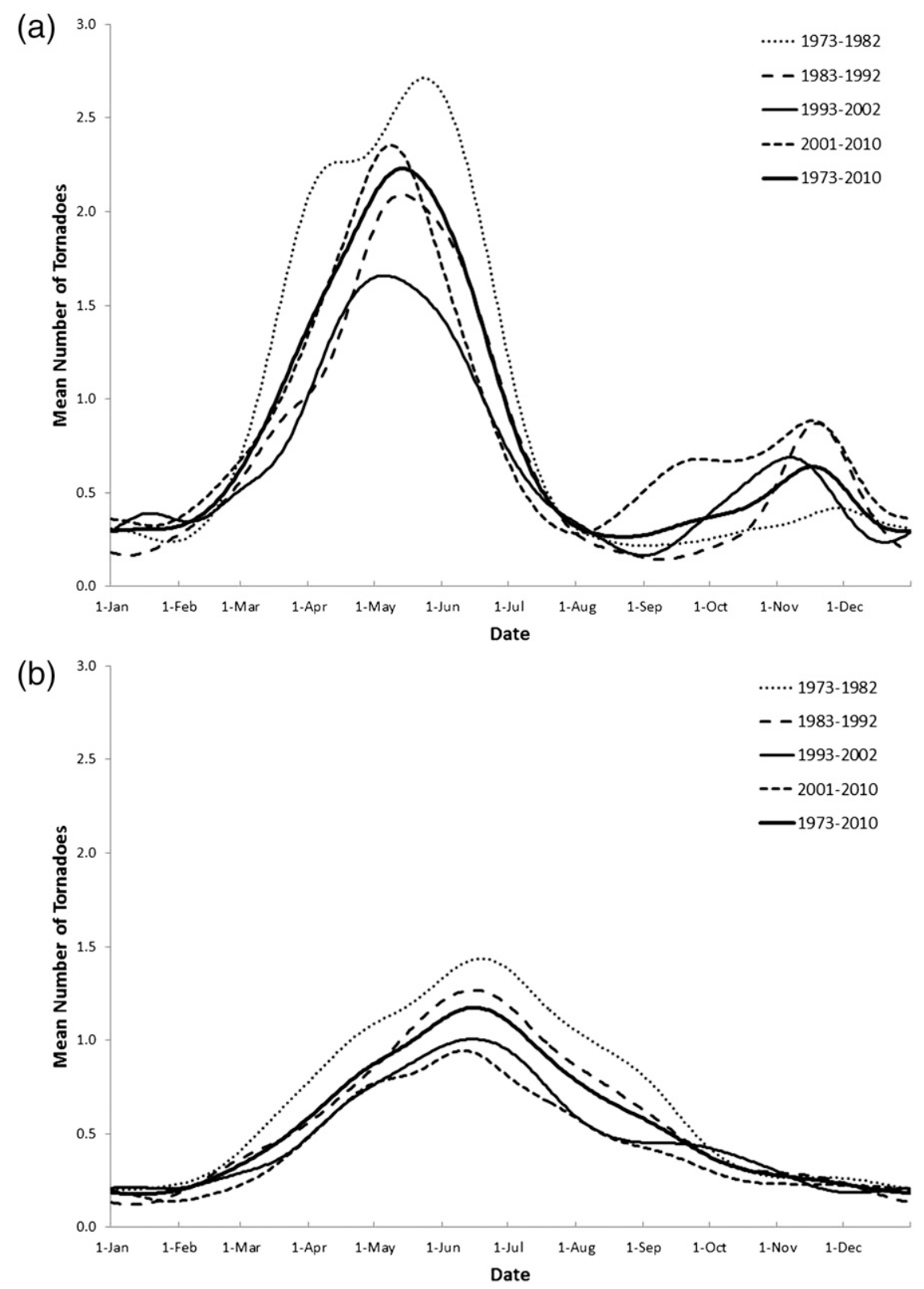

FIG. 6. As in Fig. 5, but for (a) all outbreak tornadoes and (b) nonoutbreak tornadoes during 10-yr subperiods (thin lines) and during the entire period of record (thick solid line).

peak from early March to mid-May, while the secondary peak in November is also apparent. In contrast, nonoutbreak tornadoes occur most frequently in midJune and are more frequent than outbreak tornadoes during the summer and fall months (July-October), though the secondary peak in November is not revealed among nonoutbreak tornadoes. The variability in the annual cycle was assessed by looking at 10 -yr subperiods of the record. The frequency of outbreak tornadoes exhibits a consistent peak in early to mid-May, while the secondary peak in November is only apparent after the first $10 \mathrm{yr}$ of the record (Fig. 6a). There is much variability in the magnitude of both peaks among the four subperiods, with no apparent secular trend. The timing of peak frequency is also consistent among nonoutbreak tornadoes, though the magnitude of the peak indicates a downward trend in nonoutbreak tornadoes over the 38-yr record (Fig. 6b). It is tempting to relate this trend to possible deficiencies in the underlying tornado data. However, we found that the number of F/EF1 and greater tornadoes classified into outbreaks (using the criteria described in section 2) increased by about 15\% from 1973 to 2010, 

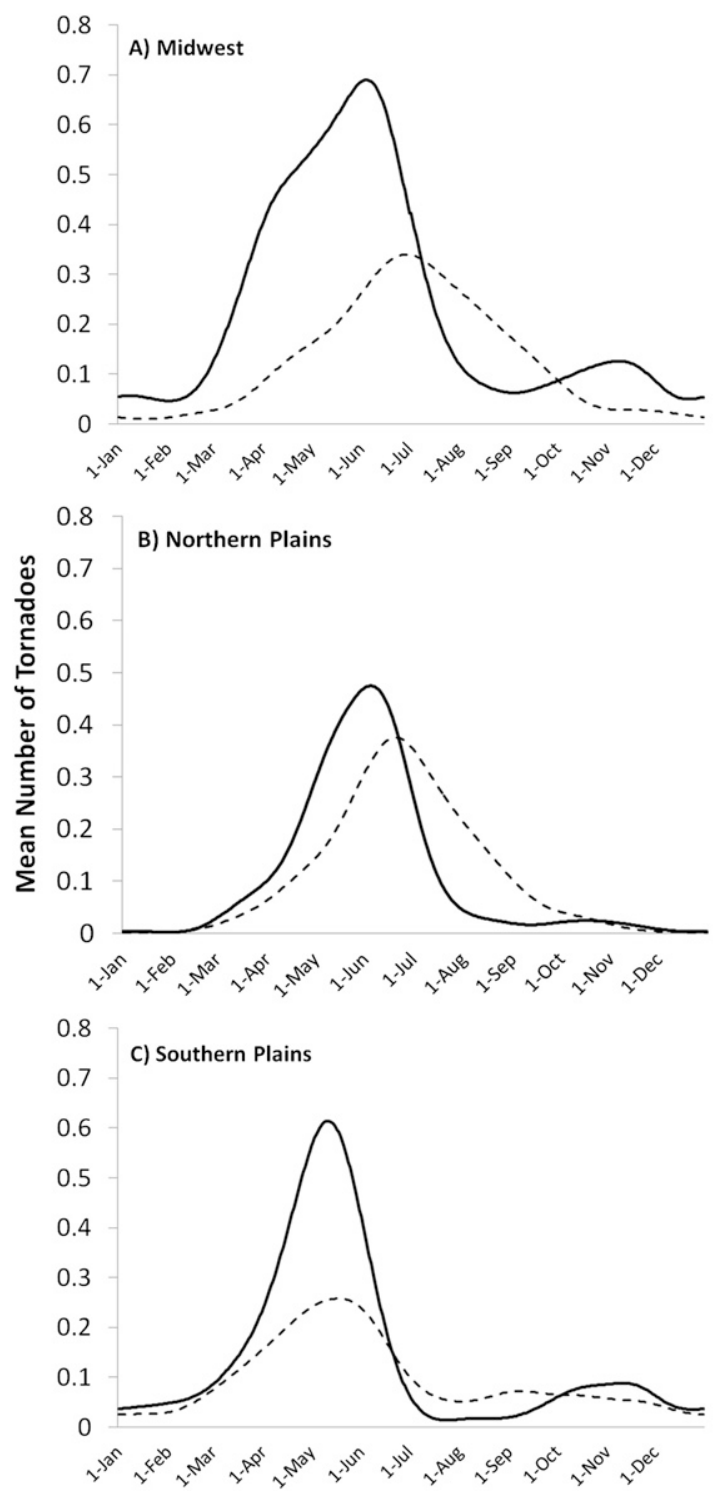

while the number classified into nonoutbreaks decreased by over $20 \%$. Since the aggregate number of F1 and greater tornadoes remained fairly stable from 1954 to 2003 (Verbout et al. 2006), and may have increased slightly since the adoption of the EF scale in 2007 (Edwards and Brooks 2010), we speculate that the apparent trend in nonoutbreak tornadoes is due to either a change in the temporal clustering of tornadoes over time (e.g., more tornadoes occurring in outbreaks), a change in the reporting and assessment of tornado damage (e.g., greater precision in demarcating individual tornado tracks), or some combination.

The spatial variability in the timing and magnitude of peak frequency among outbreak and nonoutbreak tornadoes was assessed by dividing the study area into
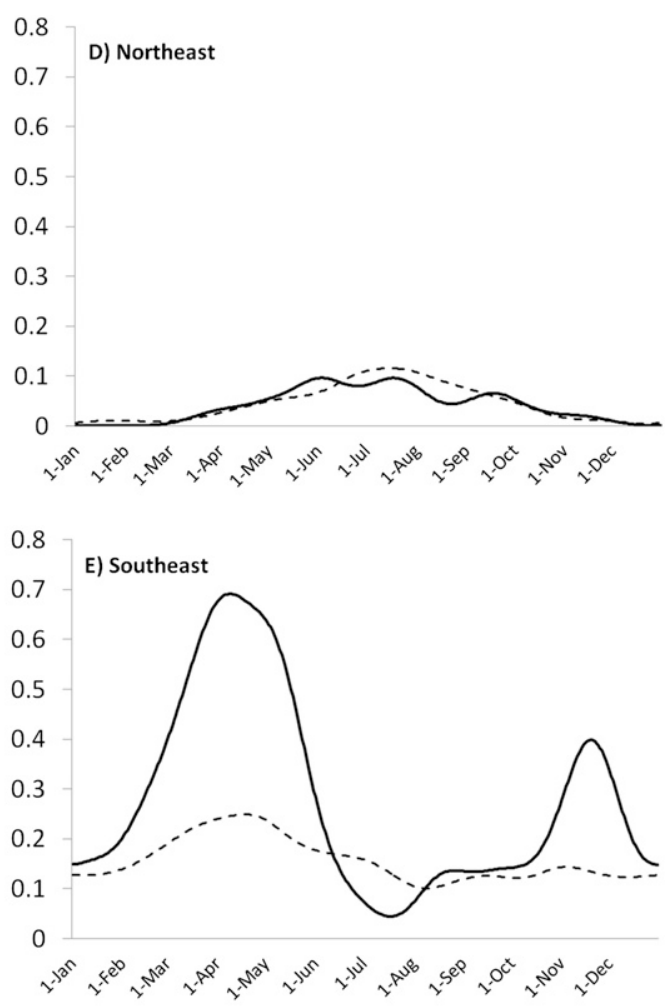

FIG. 7. Mean daily distribution of tornadoes associated with all outbreaks (solid line) and nonoutbreaks (dashed line) across five regions from 1973 to 2010: (a) Midwest $=$ MN, WI, MI, IA, IL, IN, OH, MO, KY; (b) northern plains = MT, ND, WY, SD, CO, NE, KS; (c) southern plains $=\mathrm{NM}$, OK, TX; (d) Northeast = ME, NH, VT, NY, MA, CT, RI, PA, NJ, DE, MD, WV, $\mathrm{VA}$; and (e) Southeast $=\mathrm{AR}, \mathrm{TN}, \mathrm{NC}, \mathrm{LA}, \mathrm{MS}, \mathrm{AL}$, GA, SC, FL. five regions (demarcated by state boundaries) and calculating annual cycles for each region (Fig. 7). Outbreak tornadoes exhibit a peak across the Southeast from early to mid-April, followed by a peak of slightly lower magnitude across the southern plains in early May. Outbreak tornadoes across the Midwest and southern plains reach a peak in early June, with the peak across the Midwest of similar magnitude to the peak observed across the Southeast in early April. The secondary peak in outbreak frequency in November is most pronounced across the Southeast, though smaller peaks are apparent across the Midwest and southern plains. In addition, the mean frequency of outbreak (and nonoutbreak) tornadoes across the Southeast remains above zero throughout the year, while tornado frequencies during the winter season 


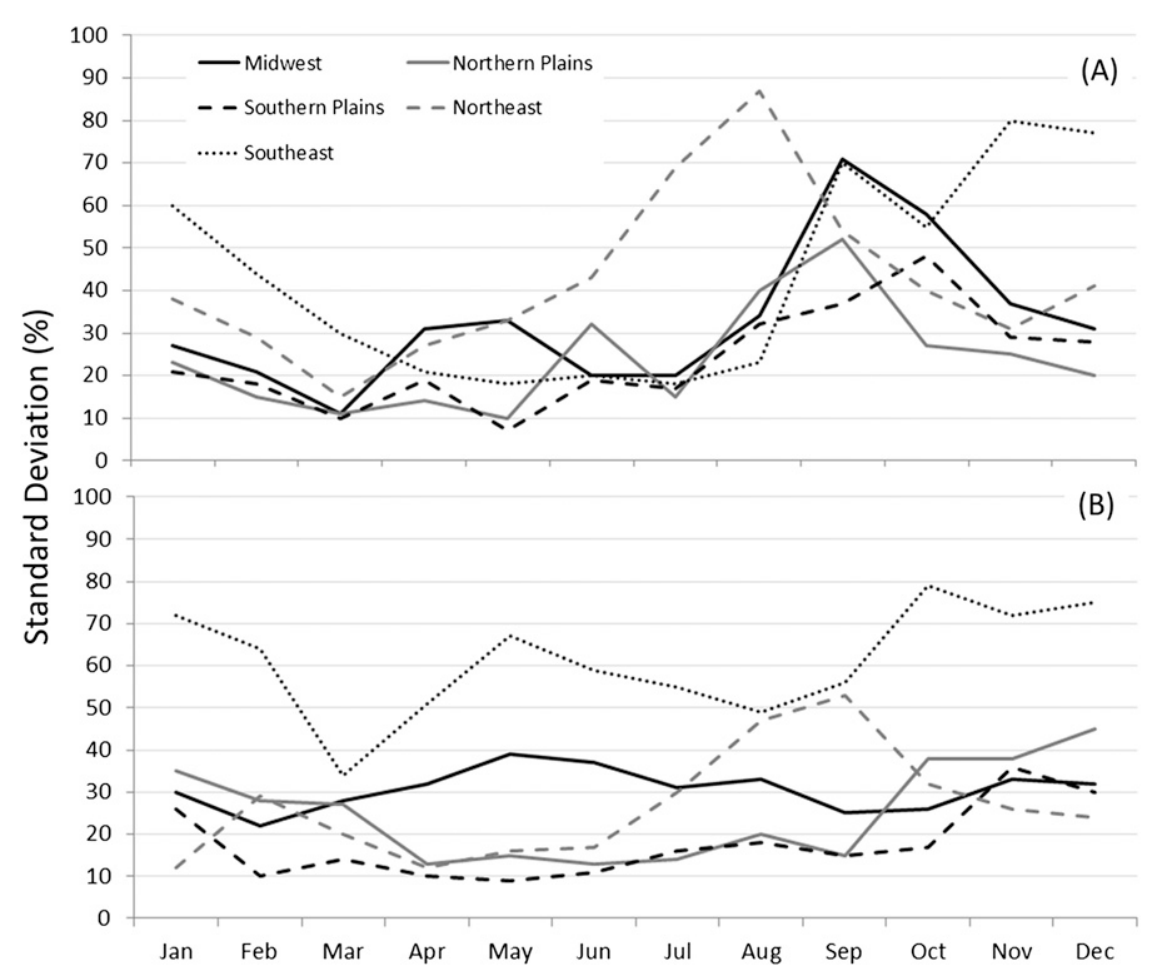

FIG. 8. Standard deviation of (a) outbreak and (b) nonoutbreak tornado counts by month for each region expressed as the percent difference from the mean monthly counts.

approach or reach zero across the other four regions. The Northeast region exhibits far fewer outbreak tornadoes than the other four regions and the majority of these tornadoes are distributed more broadly across the warm season (May-September). Nonoutbreak tornadoes exhibit well-defined peaks across the southern and northern plains and the Midwest, and these peaks occur several weeks after the peaks in outbreak tornadoes. Across the northern plains, the peak in nonoutbreak tornadoes is only slightly less than the peak in outbreak tornadoes, while across the Northeast the peak in nonoutbreak tornadoes is similar in magnitude to the peak in outbreak tornadoes. Nonoutbreak tornadoes are more broadly distributed throughout the year across the Southeast, with somewhat greater frequencies during the spring season.

The interannual variability in the occurrence of outbreak and nonoutbreak tornadoes was determined by calculating the standard deviation of monthly tornado frequencies by region, which is expressed here as a percentage of the mean frequency to help standardize for differences in tornado frequencies across months and regions (Fig. 8). With the exception of the Northeast, the relative variability in outbreak tornadoes is lowest during the spring and early summer and highest during the winter. The lower variability illustrates the year-to-year consistency in outbreak frequency during the spring severe weather season across much of the United States. Regionally, the lowest variability is found across the northern and southern plains, while the greatest variability is found across the Northeast and Southeast. These regional patterns are also evident among nonoutbreak tornadoes; however, seasonal patterns in variability are less pronounced compared to outbreak tornadoes. Overall, these results are similar to those of Brooks et al. (2003), who found that the timing (i.e., seasonality) of peak tornado frequency was better defined (i.e., less variable) across the Great Plains than across the southern and eastern United States.

\section{Tornado outbreak rankings}

One of the goals of this study was to develop a measure of tornado outbreak strength that could be easily calculated and understood as well as to provide a robust estimate of the potential lethality of outbreaks. However, for such a measure to be useful, it must yield rankings of outbreak strength that are generally consistent with what others in the severe weather community would agree upon based on either knowledge of the event or through the use of various ranking schemes (Doswell et al. 2006). In this section, we compare the rankings of the strongest 
TABLE 5. Ranking of the top-20 tornado outbreaks from 1973 to 2011 based on AFMs (at least 1000) and their ranking based on previous studies and the DPI. Cells with an asterisk represent outbreaks that were not listed in the rankings of those studies, while empty cells represent outbreaks that fell outside the study period of those studies (see footnotes below). Cells with multiple rankings indicate the rankings for individual days within multiday outbreaks. Rankings listed from previous studies and the DPI are based only on those outbreaks that occurred since 1973. Outbreaks from 2011 are set in boldface.

\begin{tabular}{|c|c|c|c|c|c|c|c|c|c|}
\hline Rank & Date & Adjusted Fujita miles & Fatalities & $\mathrm{D} 06^{\mathrm{a}}$ & V06 ${ }^{\mathrm{b}}$ & $\mathrm{FOI}^{\mathrm{c}}$ & $\mathrm{SD}_{11}{ }^{\mathrm{d}}$ & $\mathrm{SD} 12^{\mathrm{e}}$ & $\mathrm{DPI}^{\mathrm{f}}$ \\
\hline 1 & 3-4 Apr 1974 & 7060.6 & 310 & 1 & 1 & 1 & 2 & 2 & 2 \\
\hline 2 & 26-28 Apr 2011 & 6694.2 & 319 & & & 2 & 1,4 & $\mathbf{1}$ & 1 \\
\hline 3 & 21-23 Nov 1992 & 2875.6 & 26 & 5,9 & 13,18 & 3 & 17,19 & 7 & 4 \\
\hline 4 & 26-28 May 1973 & 2060.3 & 22 & 10 & $*$ & 5 & 30,66 & 16 & 19 \\
\hline 5 & 13-14 Mar 1990 & 1964.4 & 2 & 3,4 & 7 & 17 & 11 & 19 & 13 \\
\hline 6 & 31 May 1985 & 1589.4 & 76 & 2 & $*$ & 10 & 8 & 17 & 7 \\
\hline 7 & 4-5 May 2003 & 1485.3 & 38 & 7 & 14 & 16 & 5 & (4) & 12 \\
\hline 8 & 24-25 May 2011 & 1434.7 & 18 & & & 22 & 9,45 & (3) & 5 \\
\hline 9 & 7-8 Jun 1984 & 1432.7 & 13 & 14 & 15 & 27 & 28 & $*$ & 53 \\
\hline 10 & 5-6 Feb 2008 & 1374.6 & 57 & & & 7 & 3 & 10 & 8 \\
\hline 11 & 2 Jun 1990 & 1367.0 & 9 & 13 & 5 & 13 & 15 & $*$ & 36 \\
\hline 12 & 10-12 Apr 1979 & 1241.2 & 58 & $*$ & $*$ & 11 & 53,94 & $*$ & 26 \\
\hline 13 & 26-27 Apr 1991 & 1229.3 & 21 & 8 & 12 & 22 & 10 & 23 & 15 \\
\hline 14 & 2-3 Apr 1982 & 1177.5 & 30 & 6 & 3 & 9 & 13 & $*$ & 44 \\
\hline 15 & $10-11$ Nov 2002 & 1175.6 & 32 & $*$ & 4 & 21 & 7 & 13 & 16 \\
\hline 16 & 21-22 Jan 1999 & 1162.9 & 9 & $*$ & 2 & 15 & 12 & $*$ & 51 \\
\hline 17 & 12 Mar 2006 & 1161.6 & 8 & & & 34 & 6 & 12 & 31 \\
\hline 18 & 27 Mar 1994 & 1080.6 & 40 & 12 & $*$ & 66 & 20 & $*$ & 6 \\
\hline 19 & 8-9 Apr 1999 & 1070.1 & 6 & 15 & 16 & 52 & 26 & * & 25 \\
\hline 20 & 25-26 Sep 1973 & 1000.2 & 3 & $*$ & $*$ & $*$ & 89 & $*$ & 188 \\
\hline
\end{tabular}

${ }^{\text {a }}$ Rankings (1-20) from Doswell et al. (2006) (D06) using the $O$ index (period of record 1970-2003).

${ }^{\mathrm{b}}$ Rankings (1-25) from Verbout et al. (2006) (V06) using the number of F1 and greater tornadoes (period of record 1954-2003).

${ }^{\mathrm{c}}$ Rankings using the Forbes outbreak index (FOI) (period of record is from pre-1950 to 2011) obtained from G. Forbes (2013, personal communication).

${ }^{\mathrm{d}}$ Rankings from Shafer and Doswell (2011) (SD11) using the N15 weighting (period of record 1960-2011).

${ }^{\mathrm{e}}$ Rankings (1-25) from Shafer and Doswell (2012) (SD12) using the N15 weighting for multiday outbreaks (period of record 1960-2011). Note that the rankings given for the 4-5 May 2003 and 24-25 May 2011 outbreaks are for the periods 29 April-7 May 2003 and 21-25 May 2011, respectively.

${ }^{\mathrm{f}}$ Rankings using the DPI equation from Thompson and Vescio (1998).

outbreaks using our definition and metric (adjusted Fujita miles, or AFMs) to those from previous studies. These include the $O$ index from Doswell et al. (2006), the Forbes outbreak index from Forbes (2006), the N15 severe weather index from Shafer and Doswell $(2011,2012)$, the big tornado-day metric from Verbout et al. (2006), and the destruction potential index from Thompson and Vescio (1998). There are some notable differences in the parameters used to develop these ranking schemes. For example, some metrics, such as the big tornado day, consider only the number of F1 and greater tornadoes, while other metrics, such as the Forbes impact index, use a weighted scheme that considers many different outbreak attributes, such as the number of killer tornadoes, number of long-track tornadoes, and the overall tornado count. In addition, other metrics, such as the N15 severe weather index, incorporate both tornadic and nontornadic variables (e.g., significant hail and high wind reports). By comparing the results of these ranking schemes for the most significant outbreaks, the role of individual attributes in assessing outbreak strength can be evaluated.

Table 5 lists the rankings of the top 20 outbreaks in the present study (i.e., those with at least 1000 AFMs), the number of fatalities reported in each of the outbreaks, and the corresponding ranking from each of the previous studies mentioned. The date of each outbreak reflects the criteria used in the present study. In some cases, the dates did not match exactly with those in the other studies. However, if the dates of two outbreaks from different studies overlapped by at least 1 day, it was reasonable to assume they were one and the same outbreak. In addition, four of the outbreaks listed in Table 5 occurred outside the study period of at least one of the previous studies and therefore did not receive a corresponding ranking (i.e., those outbreaks with empty cells in Table 5). Two of these outbreaks occurred in 2011. The outbreak on 24-25 May 2011 extended from the Great Plains to the Ohio River valley and included several long-track and violent tornadoes, most notably 
the Hinton-Guthrie, Oklahoma, EF5 tornado (the first F/EF5 tornado in Oklahoma in over $12 \mathrm{yr}$ ), resulting in a top-10 ranking in the present study. The outbreak on 26-28 April will be discussed below. Two of the remaining three outbreaks from 2011 (15 April and 16 April; see Fig. 1) ranked in the top 25, while the 22 May outbreak, which included the Joplin, Missouri, EF5 tornado, exhibited a top-75 ranking.

Approximately half of the outbreaks listed in Table 5 yielded consistent rankings among all of the studies. Most of these outbreaks contained several moderate to strong tornadoes with at least one long-track (i.e., greater than $100 \mathrm{mi}$ or $161 \mathrm{~km})$, violent tornado $(\mathrm{F} / \mathrm{EF} 4$ or F/EF5). The strongest outbreaks exhibited numerous long-track, violent tornadoes (e.g., 3-4 April 1974, 31 May 1985, 21-23 November 1992, and 4-5 May 2003), while other outbreaks exhibited just one or a few notable tornadoes (e.g., 2-3 April 1982, Broken Bow, Oklahoma, F5 tornado; 7-8 June 1984, Barneveld, Wisconsin, F5 tornado; 13-14 March 1990, Hesston-Goessel, Kansas, F5 tornado; 26-27 April 1991, Andover, Kansas, F5 tornado). The strongest outbreaks were also generally widespread, including some that impacted several states (e.g., 26-27 May 1973 outbreak, which covered 17 states across the midwestern and southern United States). The strength and lethality of the 3-4 April 1974 and 26-28 April 2011 outbreaks are particularly noteworthy, though some differences do exist. The April 1974 outbreak exhibited an unusually large number of long-track, violent tornadoes over a relatively short amount of time [i.e., 30 tornadoes with an F4 or F5 rating over a 20-h period; Locatelli et al. (2002)], while the April 2011 outbreak exhibited more tornadoes of F/EF1 and greater strength and had a total pathlength over $300 \mathrm{~km}$ longer than the April 1974 outbreak (Knupp et al. 2014).

While there was much general agreement among the studies regarding the ranks of the strongest outbreaks, there were also notable exceptions. In some cases, an outbreak with a high number of AFMs exhibited very few tornadoes overall or several weak tornadoes and was therefore not ranked highly in some of the studies. However, of those tornadoes that did form, most were generally long track and violent. For example, only 13 tornadoes were reported in the 25-26 September 1973 outbreak, but six of those were rated F3 and greater, with two tornadoes exhibiting pathlengths of over $100 \mathrm{mi}$ $(161 \mathrm{~km})$ across parts of Oklahoma and Kansas. In other cases, differences in the methods used to define outbreaks had a significant bearing on the results. For instance, studies that used the "tornado day" metric considered consecutive days of tornado activity separately, while consecutive days of tornado activity would be considered as one outbreak in the present study if there was less than a 6-h period of tornado-free activity. An example of this discrepancy was seen in the Red River outbreak, which produced several strong and violent tornadoes on both 10 and 11 April 1979, including the Wichita Falls, Texas, F4 tornado on 10 April that killed 42 people. It is important to note that if an outbreak was not listed in one of the previous studies, it was deemed an unranked event even though it may have been ranked just outside the listed outbreaks (e.g., the outbreak was ranked 25 in the study though only the top 20 outbreaks were listed).

Although a strong relationship was identified between outbreak strength (i.e., adjusted Fujita miles) and the number of fatalities (Table 2), there were some outbreaks that resulted in a significant number of fatalities despite exhibiting relatively few AFMs. Most of these outbreaks were associated with a single violent, though not necessarily long-track, tornado that occurred among mostly weak tornadoes and tracked through highly populated areas. Examples include the 27 May 1997 Jarrell, Texas, F5 tornado (27 fatalities and 97 AFMs), and the 3 May 1999 Oklahoma City-Moore County, Oklahoma, F5 tornado (40 fatalities and $889 \mathrm{AFMs}$ ). Some of these events ranked highly in other studies (e.g., the 3-4 May 1999 event, which ranked 22nd in the present study, was ranked 5th using the Forbes outbreak index, likely due to the extraordinary financial costs). Ranking schemes that consider only the number of reported tornadoes, or emphasize other attributes such as path width and damage amount, can produce results that differ from our scheme, which emphasizes the strength of the tornadoes integrated across the pathlength. For example, over 170 tornadoes were reported across the Great Plains and Midwest from 29 to 31 May 2004, including an F4 tornado with a maximum reported width of nearly $2.5 \mathrm{mi}$ $(4 \mathrm{~km})$. As a result, this outbreak was ranked 13th when using the Forbes outbreak index but ranked 63rd in the present study due to the fact that the vast majority of the tornadoes were weak and short lived. Similarly, a total of 50 tornadoes of $\mathrm{F} 1$ and greater strength were reported across the upper Midwest on 16 June 1992, which ranked as the sixth largest big tornado day according to Verbout et al. (2006). However, the longest pathlength recorded among these tornadoes was $16 \mathrm{mi}$ $(26 \mathrm{~km})$ and therefore the outbreak received a ranking of only 67th (577 AFMs) in the present study. Additionally, because we only consider outbreaks since 1973, several well-documented and deadly outbreaks were omitted from the analysis, including the 1965 Palm Sunday outbreak (over 270 fatalities) and the 1971 Mississippi Delta outbreak (over 120 fatalities).

Tornado outbreaks may also be spawned by landfalling tropical cyclones. Verbout et al. (2007) used the 
big-tornado-day metric to identify outbreaks associated with tropical cyclones that made landfall along the U.S. coast from 1954 to 2004. Most of these outbreaks consisted of weak (F0 and F1) and short-lived tornadoes resulting from tropical cyclones that made landfall along the northern Gulf Coast. Of the 34 tornado-producing tropical cyclones from 1973 to 2004 identified in Verbout et al. (2007), only 4 spawned outbreaks with at least 100 AFMs. The strongest outbreak according to our scheme was spawned by Hurricane Danny in August 1985 with 359 AFMs, followed by Hurricane Ivan in September 2004 with 275 AFMs. Although both of these tropical cyclones spawned over 100 tornadoes, more than $80 \%$ were weak and short lived, therefore resulting in relatively few AFMs.

\section{Discussion and conclusions}

In this study, we developed two related metrics (hectopascal miles and Fujita miles) to measure the physical magnitude, or strength, of tornado outbreaks east of the Rocky Mountains. To account for variations in tornado strength along a given track, we calculated adjusted forms of these metrics. The simpler metric, adjusted Fujita miles (AFMs), was used to rank tornado outbreaks and examine their climatological and geographical characteristics. Comparisons were also made between the results of our ranking scheme and those of previous studies. Finally, we assessed how the strongest outbreaks of 2011 ranked among all outbreaks in the modern record (1973-2010). Although there is no one best approach to defining and ranking tornado outbreaks, our scheme accounts for nonmeteorological trends in the data, is reproducible, and yields rankings generally consistent with what others would arrive at subjectively (Doswell et al. 2006). While ranking schemes that incorporate multiple variables may be considered more robust than schemes that use a single combined variable (as in this study), our scheme was intended to quantify the physical strength of outbreaks and assess the relationship between the overall risk (i.e., exposure) associated with outbreaks and the resulting number of injuries and fatalities. This relationship cannot be assessed using multivariate schemes that incorporate injuries and fatalities as independent variables, such as those described in Doswell et al. (2006) and Shafer and Doswell $(2010,2011)$. Moreover, ranking schemes that include societal impacts are subject to the vagaries of where tornadoes happen to occur (e.g., highly populated versus sparsely populated areas). Nevertheless, we emphasize that this study is not attempting to establish a universal definition or ranking scheme for tornado outbreaks. Indeed, even minor changes in the criteria used to define an outbreak, such as the time between consecutive tornadoes in a sequence, will have some bearing on the relative rankings.

Similar to previous studies (e.g., Galway 1977; Schneider et al. 2004), we found that the vast majority of tornado-related fatalities (approximately $80 \%$ ) occurred in outbreaks. In a given year, most tornado outbreaks will be relatively weak and result in only a few casualties. However, some outbreaks can be quite strong and involve several violent, long-track tornadoes. For instance, although outbreaks with at least 1000 AFMs accounted for less than $3 \%$ of all outbreaks, they were responsible for over one-third of all violent tornadoes and over $30 \%$ of all tornado-related fatalities. Additionally, the range in strength among the strongest outbreaks was quite considerable compared to the rest of the outbreaks, which supports the findings of previous studies that employed more sophisticated multivariate ranking schemes to identify major tornado outbreaks (Shafer and Doswell 2010, 2011). Using the Fujita miles metric, we noted a strong positive relationship between outbreak strength and the number of fatalities and injuries. This relationship was stronger than that using other measures of outbreak strength, including the destruction potential index and the number of F/EF2 and greater tornadoes, suggesting that the Fujita miles metric is more reflective of the potential threat of outbreaks to human life. Therefore, a ranking scheme based on Fujita miles may be useful to forecasters as guidance for categorizing the threat level for tornadoes [e.g., high, moderate, and slight risk; see Shafer and Doswell (2010)]. An evaluation of the utility of Fujita miles in an operational setting is left to future work.

We noted climatological differences in the spatial and temporal distributions of outbreak and nonoutbreak tornadoes. The risk from outbreak tornadoes was greatest across the lower Mississippi River valley, where significant tornadoes are most common (see Figs. 6, 8, and 11 in Coleman and Dixon 2014). Coleman and Dixon (2014) also found this area to be at greatest risk for significant tornado pathlengths, although the area of maximum risk in their study extended farther east into Alabama. The number of killer tornadoes is also greatest in this region (Ashley 2007). Taken together, the results of previous work and the present study suggest that the Deep South is at greatest risk from strong, long-track, killer tornadoes, and that these tornadoes are most likely to occur in outbreaks. Nonoutbreak tornadoes also occurred frequently across the lower Mississippi River valley, with a secondary maximum across part of the upper Midwest during the summer months. Outbreak tornadoes typically occurred earlier in the spring season than nonoutbreak tornadoes and were responsible for the secondary peak in tornado 
frequency that has been documented in the fall season, particularly across the Southeast (Brooks et al. 2003). Because outbreak tornadoes are clustered in time (and presumably in space), it is likely that they may be associated with discrete synoptic-scale circulation patterns, which could offer forecasters improved guidance and predictability over nonoutbreak tornadoes. We intend to examine this hypothesis in a follow-up study. Due to the short 38-yr period of record used in the present study, long-term trends in the frequency of outbreak and nonoutbreak tornadoes could not be assessed (Doswell 2007). Future work may consider using data compiled by Grazulis (1993) for F2 and greater tornadoes extending back to 1916 to identify relationships between outbreak strength and climate variability. In addition, more research is needed to understand the trends in outbreak and nonoutbreak tornadoes, particularly over the past few decades, as shown in this study. Such information could help determine whether there has been a real change in the behavior of tornadoes (e.g., an increasing number of isolated tornadoes or smaller, weaker outbreaks) or whether the trends are artificial (e.g., changes in reporting practices and damage assessments).

We attempted to develop a ranking scheme that relates the physical magnitude, or strength, of an outbreak to the number of fatalities and injuries. Although there can be large differences between the meteorological significance of an outbreak and its societal impact, the potential for destruction and loss of life (i.e., risk) is greatest where exposure and intensity are maximized. Our measure of outbreak strength (Fujita miles) emphasizes the cumulative risk associated with exposure to tornadoes of varying intensity (i.e., pathlength and $\mathrm{F} / \mathrm{EF}$-scale rating) integrated across all tornadoes in an outbreak. The strong correlation between Fujita miles and the number of fatalities and injuries suggests that our measure of outbreak strength provides a reasonable assessment of the potential lethality of outbreaks. However, we are not claiming that the Fujita miles metric is the best way to rank the significance of tornado outbreaks. Indeed, in some cases a particularly strong outbreak resulted in relatively few fatalities (e.g., the March 1990 Great Plains outbreak; Table 5), while outbreaks consisting of only one or a few strong to violent tornadoes may not have been ranked particularly high but still resulted in significant damage and/or loss of life (e.g., the outbreak associated with the Greensburg, Kansas, EF5 tornado in May 2007).

To assess how the use of different attributes affected the ranking of tornado outbreaks, we compared the results of our ranking scheme for the top-20 outbreaks to those of previous studies. We noted that the strongest outbreaks generally yielded consistent rankings among all of the studies. These outbreaks were of long duration (i.e., at least 1 day); exhibited numerous tornadoes, including several that were violent and long-track; and had high numbers of casualties and significant property damage. Therefore, the strongest and most destructive outbreaks are likely to be ranked highly regardless of the specific criteria. However, ranking schemes that consider only the overall number of tornadoes (with no emphasis placed on F/EF-scale rating) or use the tornado-day metric are more likely to produce varying results. For example, an outbreak consisting of numerous weak tornadoes may be ranked higher than an outbreak with just a few strong to violent tornadoes. In this case, a multivariate index that accounts for tornado frequency and intensity can provide a more robust measure of outbreak strength (Doswell et al. 2006). In addition, using the tornado-day metric, a period of generally continuous tornado activity that extends beyond $24 \mathrm{~h}$ will be considered as multiple outbreaks, and this may have some bearing on the relative rankings (Shafer and Doswell 2012). We also note that there was much variability between our rankings and those computed using the destruction potential index. As noted in section 2, the reporting of path width (which is used in the calculation of the index) is not consistent in the historical tornado database, although it is unclear what impact this had on the rankings.

The present work was primarily motivated by the tornado outbreaks of 2011, which contributed to the deadliest tornado year in the United States in 75 years. Most of the tornado-related fatalities in 2011 occurred across the southern United States from 26 to 28 April in an outbreak that has drawn comparisons to the April 1974 Super Outbreak. Based on the Fujita miles metric for ranking outbreaks by strength, we find these comparisons to be valid, as both outbreaks ranked well above the next strongest outbreaks in the historical record since 1973 (Fig. 3). While quantitative measures of outbreak strength will likely rank one outbreak as stronger than the other (e.g., Shafer and Doswell 2011, 2012; Doswell et al. 2012; Knupp et al. 2014; Forbes impact index, G. Forbes 2013, personal communication), those differences are insignificant when considering the nature of tornado data collection and, perhaps most importantly, the resulting impacts to society and human life. Despite improvements in the lead times of tornado warnings and the ability to disseminate information to a wider segment of the population in the 37 years between these two outbreaks, the events of 2011 reminded us that major tornado outbreaks, particularly those with violent and long-track tornadoes, can still result in significant destruction and loss of life. Reducing our vulnerability will require a better understanding of the relationships 
between the physical and societal aspects of tornado outbreaks.

Acknowledgments. We thank Greg Carbin of NOAA's Storm Prediction Center for his insights on the tornado database and for his comments on an earlier version of this study. We also thank Stuart Hinson of NOAA's National Climatic Data Center and Brent Macaloney of NOAA's National Weather Service for providing access to the Performance Management and Data Verification website. We greatly appreciate the efforts of Dr. Greg Forbes, Dr. Chad Shafer, and one anonymous reviewer, whose comments and suggestions significantly improved the manuscript. We also thank Drs. Forbes and Shafer for sharing their updated outbreak rankings. Funding for this project was provided by NOAA as part of the Regional Climate Center Program.

\section{REFERENCES}

Ashley, W. S., 2007: Spatial and temporal analysis of tornado fatalities in the United States: 1880-2005. Wea. Forecasting, 22, 1214-1228, doi:10.1175/2007WAF2007004.1.

Boruff, B. J., J. A. Easoz, S. D. Jones, H. R. Landry, J. D. Mitchem, and S. L. Cutter, 2003: Tornado hazards in the United States. Climate Res., 24, 103-117, doi:10.3354/cr024103.

Brooks, H. E., 2004: On the relationship of tornado path length and width to intensity. Wea. Forecasting, 19, 310-319, doi:10.1175/ 1520-0434(2004)019<0310:OTROTP > 2.0.CO;2.

—_, and C. A. Doswell III, 2001: Normalized damage from major tornadoes in the United States: 1890-1999. Wea. Forecasting, 16, 168-176, doi:10.1175/1520-0434(2001)016<0168: NDFMTI $>2.0 . \mathrm{CO} ; 2$

- and J. P. Craven, 2002: A database of proximity soundings for significant severe thunderstorms: 1957-1993. Preprints, 21st Conf. on Severe Local Storms, San Antonio, TX, Amer. Meteor. Soc., 16.2. [Available online at https://ams.confex.com/ ams/pdfpapers/46680.pdf.]

—, C. A. Doswell III, and M. Kay, 2003: Climatological estimates of local daily tornado probability for the United States. Wea. Forecasting, 18, 626-640, doi:10.1175/1520-0434(2003)018<0626: CEOLDT $>2.0 . \mathrm{CO} ; 2$.

Coleman, T. A., and P. G. Dixon, 2014: An objective analysis of tornado risk in the United States. Wea. Forecasting, 29, 366-376. doi:10.1175/WAF-D-13-00057.1.

Concannon, P. R., H. E. Brooks, and C. A. Doswell, 2000: Climatological risk of strong and violent tornadoes in the United States. Preprints, Second Symp. on Environmental Applications, Long Beach, CA, Amer. Meteor. Soc., 9a. [Available online at https://ams.confex.com/ams/annual2000/webprogram/ Paper6471.html.]

Dixon, P. G., A. E. Mercer, J. Choi, and J. S. Allen, 2011: Tornado risk analysis: Is Dixie Alley an extension of Tornado Alley? Bull. Amer. Meteor. Soc., 92, 433-441, doi:10.1175/2010BAMS3102.1.

,,-- K. Grala, and W. H. Cooke, 2014: Objective identification of tornado seasons and ideal spatial smoothing radii. Earth Interact., 18, doi:10.1175/2013EI000559.1.

Doswell, C. A., III, 2007: Small sample size and data quality issues illustrated using tornado occurrence data. Electron. J. Severe
Storms Meteor., 2 (5). [Available online at http://www.ejssm. org/ojs/index.php/ejssm/article/viewarticle/26/27.]

— tornado climatology. Mon. Wea. Rev., 116, 495-501, doi:10.1175/ 1520-0493(1988)116<0495:OSIOUS > 2.0.CO;2.

— R. Edwards, R. L. Thompson, J. A. Hart, and K. C. Crosbie, 2006: A simple and flexible method for ranking severe weather events. Wea. Forecasting, 21, 939-951, doi:10.1175/WAF959.1.

- H. E. Brooks, and N. Dotzek, 2009: On the implementation of the enhanced Fujita scale in the USA. Atmos. Res., 93, 554563, doi:10.1016/j.atmosres.2008.11.003.

— G. W. Carbin, and H. E. Brooks, 2012: The tornadoes of spring 2011 in the USA: An historical perspective. Weather, 67, 88-94, doi:10.1002/wea.1902.

Edwards, R., and H. E. Brooks, 2010: Possible impacts of the enhanced Fujita scale on United States tornado data. Preprints, 25th Conf. on Severe Local Storms, Denver, CO, Amer. Meteor. Soc., P8.28. [Available online at https://ams.confex.com/ ams/pdfpapers/175398.pdf.]

— J. G. LaDue, J. T. Ferree, K. Scharfenberg, C. Maier, and W. L. Coulbourne, 2013: Tornado intensity estimation: Past, present, and future. Bull. Amer. Meteor. Soc., 94, 641-653, doi:10.1175/BAMS-D-11-00006.1.

Forbes, G. S., 2006: Meteorological aspects of high-impact tornado outbreaks. Preprints, Symp. on the Challenges of Severe Convective Storms, Atlanta, GA, Amer. Meteor. Soc., P1.12. [Available online at https://ams.confex.com/ams/pdfpapers/ 99383.pdf.]

Galway, J. G., 1977: Some climatological aspects of tornado outbreaks. Mon. Wea. Rev., 105, 477-484, doi:10.1175/ 1520-0493(1977)105<0477:SCAOTO >2.0.CO;2.

Grazulis, T. P., 1993: Significant Tornadoes 1680-1991. Environmental Films, 1326 pp.

Hagemeyer, B. C., 1997: Peninsular Florida tornado outbreaks. Wea. Forecasting, 12, 399-427, doi:10.1175/1520-0434(1997)012<0399: PFTO $>2.0 . \mathrm{CO} ; 2$.

Hamill, T. M., R. S. Schneider, H. E. Brooks, G. S. Forbes, H. B. Bluestein, M. Steinberg, D. Melendez, and R. M. Dole, 2005: The May 2003 extended tornado outbreak. Bull. Amer. Meteor. Soc., 86, 531-542, doi:10.1175/BAMS-86-4-531.

Johns, R. H., and W. R. Sammler, 1989: A preliminary synoptic climatology of violent tornado outbreaks utilizing radiosonde standard level data. Preprints, 12th Conf. on Weather Analysis and Forecasting, Monterey, CA, Amer. Meteor. Soc., 196-201.

Kay, M. P., and H. E. Brooks, 2000: Verification of probabilistic severe storm forecasts at the SPC. Preprints, 20th Conf. on Severe Local Storms, Orlando, FL, Amer. Meteor. Soc., 9.3. [Available online at https://ams.confex.com/ams/pdfpapers/ 15921.pdf.]

Knupp, K. R., and Coauthors, 2014: Meteorological overview of the devastating 27 April 2011 tornado outbreak. Bull. Amer. Meteor. Soc., doi:10.1175/BAMS-D-11-00229.1, in press.

Locatelli, J. D., M. T. Stoelinga, and P. V. Hobbs, 2002: A new look at the Super Outbreak of tornadoes on 3-4 April 1974. Mon. Wea. Rev., 130, 1633-1651, doi:10.1175/1520-0493(2002)130<1633: ANLATS $>2.0 . \mathrm{CO} ; 2$.

Mercer, A. E., C. M. Shafer, C. A. Doswell III, L. M. Leslie, and M. B. Richman, 2009: Objective classification of tornadic and nontornadic severe weather outbreaks. Mon. Wea. Rev., 137, 4355-4368, doi:10.1175/2009MWR2897.1.

National Oceanic and Atmospheric Administration cited 2012: 2011 tornado information. [Available online at http://www. noaanews.noaa.gov/2011_tornado_information.html.] 
O'Sullivan, D., and D. J. Unwin, 2003: Geographic Information Analysis. John Wiley and Sons, $436 \mathrm{pp}$.

Pautz, M. E., 1969: Severe local storm occurrences, 1955-1967. ESSA Tech. Memo. WBTM FCST12, 77 pp.

Schneider, R. S., H. E. Brooks, and J. T. Schaefer, 2004: Tornado outbreak days: An updated and expanded climatology (18752003). Preprints, 22nd Conf. on Severe Local Storms, Hyannis, MA, Amer. Meteor. Soc., P5.1. [Available online at https:// ams.confex.com/ams/pdfpapers/82031.pdf.]

Shafer, C. M., and C. A. Doswell III, 2010: A multivariate index for ranking and classifying severe weather outbreaks. Electron. J. Severe Storms Meteor., 5 (1). [Available online at http:// ejssm.org/ojs/index.php/ejssm/article/viewArticle/62.]

__ and _ 2011: Using kernel density estimation to identify, rank, and classify severe weather outbreak events. Electron. J. Severe Storms Meteor., 6 (2). [Available online at http:// www.ejssm.org/ojs/index.php/ejssm/article/view/74/.]

- and - 2012: Identifying and ranking multi-day severe weather outbreaks. Preprints, 26th Conf. on Severe Local Storms, Nashville, TN, Amer. Meteor. Soc., 77. [Available online at https://ams.confex.com/ams/26SLS/webprogram/ Paper211637.html.]
Smith, B. T., R. L. Thompson, J. S. Grams, C. Broyles, and H. E. Brooks, 2012: Convective modes for significant severe thunderstorms in the contiguous United States. Part I: Storm classification and climatology. Wea. Forecasting, 27, 11141135, doi:10.1175/WAF-D-11-00115.1.

Stimers, M., 2012: Viewing tornado impact as more than physical magnitude. Weatherwise, 65, 18-24, doi:10.1080/ 00431672.2012.689587.

Storm Prediction Center, cited 2013: Annual U.S. killer tornado statistics, 2011. [Available online at http://www.spc.noaa.gov/ climo/torn/fatalmap.php?yr=2011.]

Thompson, R. L., and M. D. Vescio, 1998: The destruction potential index - A method for comparing tornado days. Preprints, 19th Conf. on Severe Local Storms, Minneapolis, MN, Amer. Meteor. Soc., 280-282.

Verbout, S. M., H. E. Brooks, L. M. Leslie, and D. M. Schultz, 2006: Evolution of the U.S. tornado database: 1954-2003. Wea. Forecasting, 21, 86-93, doi:10.1175/WAF910.1.

D. M. Schultz, L. M. Leslie, H. E. Brooks, D. J. Karoly, and K. L. Elmore, 2007: Tornado outbreaks associated with landfalling hurricanes in the North Atlantic basin: 1954-2004. Meteor. Atmos. Phys., 97, 255-271, doi:10.1007/s00703-006-0256-x. 